\title{
Intraocular pressure, systemic blood pressure, and age: a correlational study
}

\author{
MICHAEL SCHULZER' AND STEPHEN M DRANCE' \\ From the 'Departments of Statistics and Medicine and the 'Department of Ophthalmology, University of British \\ Columbia, Vancouver, Canada
}

SUMmaRY An attempt was made to study the associations between age, intraocular pressure, and systolic blood pressure. The eyes of 90 non-glaucomatous, ocular normotensive patients and of 142 ocular hypertensive patients were examined. A third group was formed from these two by stratification, to mirror the intraocular pressure distribution in the general population. In all three groups systolic blood pressure was positively correlated with age. In the stratified group a 'chain' model was consistent with the data. Intraocular pressure was significantly negatively correlated with systolic blood pressure; any apparent association between intraocular pressure and age was fully accounted for through the correlation between systolic blood pressure and age.

The relationship between intraocular pressure, age, and various cardiovascular risk variables is not fully understood. The most significantly correlated triad appears to be intraocular pressure, age, and blood pressure, but the associations between these three variables have been inconsistently reported.

Several univariate studies seem to indicate significant positive correlations between intraocular pressure (IOP) and age, and IOP and blood pressure (BP). ${ }^{1-3}$ Klein and Klein' refer to the possible interference of age as it affects the association they observe between IOP and BP.

Leske and Podgor ${ }^{+}$examined 2315 eyes and carried out a stepwise regression of IOP on a large carrier set, including age, sex, height, systolic and diastolic BP, diabetes, ventricular rate, and vertical and horizontal cup/disc ratio. They found a positive correlation of IOP with systolic BP $\left(R^{2}=3 \%\right)$; with other, less significant variables added to the regression, the final $\mathrm{R}^{2}$ reached $5 \cdot 8 \%$. Age was found not to be significant once systolic BP had entered the regression. They also made the interesting observation that IOP and systolic BP seemed to be correlated only in eyes with glaucomatous visual defects; moreover, the ratio of systolic BP to IOP was significantly lower in eyes which had such defects. They suggested that the nonsignificance of age may be attributable to the hypo-

Correspondence to Dr Michael Schulzer. Department of Statistics, University of British Columbia, 2075 Wesbrook Mall, Vancouver, BC. Canada V6T 1 W5. thesis that, after controlling for BP, the age effect on IOP may be present only in younger persons. They further raised the possibility that raised systemic pressure may precede raised IOP in a given patient, but did not test this with their cross-sectional data.

Shiose et al. ${ }^{56}$ examined 127842 eyes. Univariate regressions indicated a negative correlation of IOP with age, a positive correlation with body weight, and a positive correlation with BP. They performed a stepwise regression of IOP on a carrier set containing BP, age, weight, calcium, packed cell volume, haemoglobin, glucose tolerance, alkaline phosphatase, and other variables, and obtained a regression with positive coefficients on weight and $\mathrm{BP}$ and a negative coefficient on age, yielding $\mathrm{R}^{2}=4 \cdot 8 \%$. They attempted to explain the negative correlation with age by suggesting that the Japanese population tends to be less hypertensive and less obese with age than Caucasian populations, which report a positive age correlation. This explanation may not, however, be sufficient, since the partial regression coefficients in a multiple regression equation are adjusted for the presence of the other covariates in the equation. In a recent study by Daoping Lu et al. ${ }^{7}$ on 155 eyes followed for a five-year period, a significant positive correlation between systolic $\mathrm{BP}$ and IOP $\left(\mathrm{R}^{2}=\right.$ $15.7 \%$ ) was found only in progressing cases of glaucoma.

The present study was undertaken with the aim of combining the use of regression and of path 
Fig. 1 Hypothetical schematic models for the age/BP/IOP relationships. A: $B P / I O P$ independence. B: All pairs correlated. C: Age/IOP conditionally independent, given $B P$.

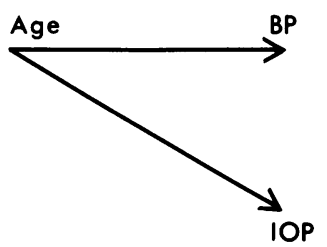

Model A

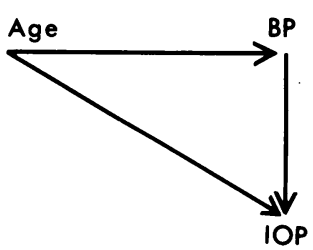

B

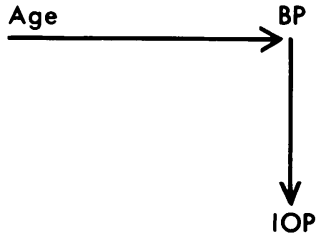

C analysis $^{x-11}$ in order to elucidate the associations among the three variables, IOP, BP, and age. Three models were examined (Fig. 1). Model A allowed for independent age effects on both BP and IOP, model $B$ considered pairwise interactions for all pairs of variables (age and BP, age and IOP, BP and IOP), and model $C$, a chain model, allowed only for age effect on BP and for a direct BP effect on IOP. It may be recalled that the chain model was recognised by Galton $^{12}$ in the 19th century, when he pointed out that, if the sole 'cause' of correlation between the traits $i$ and $j$ was a third trait $g$, then the relationship

$$
r_{i j}=r_{i g} r_{i g}
$$

must hold between the pairwise correlation coefficients. This was later validated by Spearman ${ }^{13} 14$ in his characterisation of 'general intelligence', and it was ultimately seen to be equivalent to a zero partial correlation coefficient.

\section{Materials and methods}

The sample consisted of 90 normal patients and 142 patients with ocular hypertension. These patients were fully described in our previous work ${ }^{1516}$ on the use of discriminant analysis in predicting glaucomatous visual field damage. The normal sample was selected to be in the same age group as our regular run of glaucoma patients, and our approach was to encourage the spouses of glaucoma patients to volunteer for the normal study. Normal patients had a full ocular examination including ophthalmodynamometry, gonioscopy, stereoexamination of the optic nerve heads; systemic blood pressures were measured and a full history taken. A neurovascular examination was carried out, excluding invasive procedures unless these were clinically indicated from a neurological standpoint. $X$-rays of the sella and optic foramina excluded those patients with obvious space occupying lesions. Laboratory tests included electrocardiography, 2-hour post-cibum blood glucose, blood urea nitrogen, uric acid concentrations, ESR, haemoglobin level, serum cholesterol and triglycerides, and a T4 test for thyroid function. The visual field had to be normal when examined by Armaly's selective perimetry ${ }^{17}$ method as modified by us $^{18}$ on the Goldmann perimeter. Intraocular pressures checked on at least two occasions had to be below $21 \mathrm{mmHg}$.

The ocular hypertensive group consisted of consecutive patients with repeated applanation pressures above $21 \mathrm{mmHg}$ in at least one eye, having normal visual fields, examined in the same way, and with normal optic nerve heads on stereoophthalmoscopy.

The associations among the three variables, age, $\mathrm{BP}$, and IOP, were examined within the normal and the ocular hypertensive groups. In addition a stratified group was formed from these two to represent a general non-glaucomatous population (93\% normal pressures, $7 \%$ ocular hypertensives), by adding a random selection of seven patients from the ocular hypertensive group to the 90 normal persons. The relationships between the variables were explored separately in the stratified group.

In an attempt to investigate these relations univariate regressions were first calculated between each pair of variables. In addition, multiple regressions of IOP on age and BP were calculated. Following the techniques of simultaneous regressions and path analysis $^{\text {t11 }}$ an attempt was made to fit the most appropriate conceptual model to each group. The models (Fig. 1) correspond to different sets of regression equations. Model $\mathrm{A}$ is represented by two simultaneous univariate equations: $\mathrm{BP}$ on age and IOP on age. Model $\mathrm{B}$, a recursive model, is repre-

Table 1 Data summary: means and standard deviations for the three groups

\begin{tabular}{|c|c|c|c|c|c|}
\hline & Age (years) & Systolic BP & Mean BP & $\begin{array}{l}\text { IOP } \\
\text { (mean of } \\
2 \text { eyes) }\end{array}$ & $\begin{array}{l}\text { Max IOP } \\
(\text { max of } \\
2 \text { eyes) }\end{array}$ \\
\hline $\begin{array}{l}\text { Normals } n=90 \\
\text { Ocular hypertensives } n=142 \\
\text { Stratified } n=97\end{array}$ & $\begin{array}{l}63 \cdot 6 \pm 12 \cdot 3 \\
59 \cdot 8 \pm 10 \cdot 3 \\
63 \cdot 7 \pm 12 \cdot 0\end{array}$ & $\begin{array}{l}142 \cdot 0 \pm 26 \cdot 4 \\
140 \cdot 7 \pm 26 \cdot 2 \\
141 \cdot 1 \pm 25 \cdot 9\end{array}$ & $\begin{array}{l}101 \cdot 9 \pm 15 \cdot 8 \\
102 \cdot 9 \pm 14 \cdot 5 \\
101 \cdot 9 \pm 15 \cdot 4\end{array}$ & $\begin{array}{l}15 \cdot 4 \pm 2 \cdot 7 \\
26 \cdot 2 \pm 3 \cdot 9 \\
16 \cdot 1 \pm 3 \cdot 8\end{array}$ & $\begin{array}{l}16 \cdot() \pm 2 \cdot 9 \\
27 \cdot 4 \pm 4 \cdot 6 \\
16 \cdot 7 \pm 3 \cdot 9\end{array}$ \\
\hline
\end{tabular}


Table 2 Joint distribution of low versus high IOP and systolic $B P^{*}$ with age in the stratified group

\begin{tabular}{clcccc}
\hline & $\begin{array}{l}\text { Low IOP. } \\
\text { low BP }\end{array}$ & $\begin{array}{l}\text { Low IOP, } \\
\text { high BP }\end{array}$ & $\begin{array}{l}\text { High IOP. } \\
\text { low BP }\end{array}$ & $\begin{array}{l}\text { High IOP. } \\
\text { high BP }\end{array}$ & Totals \\
\hline Agc 54 and & 4 & 2 & 8 & 3 & 17 \\
younger & $0 \cdot 3+$ & $-1 \cdot 7$ & $2 \cdot 3$ & $-0 \cdot 8$ & \\
$55-64$ & 8 & 7 & 9 & 8 & 32 \\
& $0 \cdot 7$ & $1 \cdot 1$ & $0 \cdot 5$ & $-0 \cdot 1$ & \\
65 and older & 8 & 19 & 7 & 14 & 48 \\
& $-1 \cdot 0$ & $2 \cdot 3$ & $-2 \cdot 3$ & $0 \cdot 8$ & \\
Totals & 20 & 28 & 24 & 25 & 97 \\
\hline
\end{tabular}

${ }^{*}$ Low IOP: less than $15 \cdot 5 \mathrm{mmHg}$. Low BP: less than $140 \mathrm{mmHg}$. $\mathbf{p}=\mathbf{0} \cdot 08$. $†$ Adjusted residual.

sented by a univariate and a bivariate regression, solved simultaneously: BP on age, and IOP on age and BP. Model C corresponds to two simultaneous univariate equations: a regression of $\mathrm{BP}$ on age and of IOP on BP.

\section{Results}

In the sequel, IOP denotes the mean of two eyes, and max IOP denotes the maximum of two eyes.

Table 1 summarises the raw data for each group. Table 2 shows the joint distribution in the stratified group of IOP and systolic BP (both dichotomised into 'above' versus 'below' group medians) across age levels. Table 2 also indicates that no statistical correlation with age could be found in the data with regard to the order of occurrence of raised systolic BP

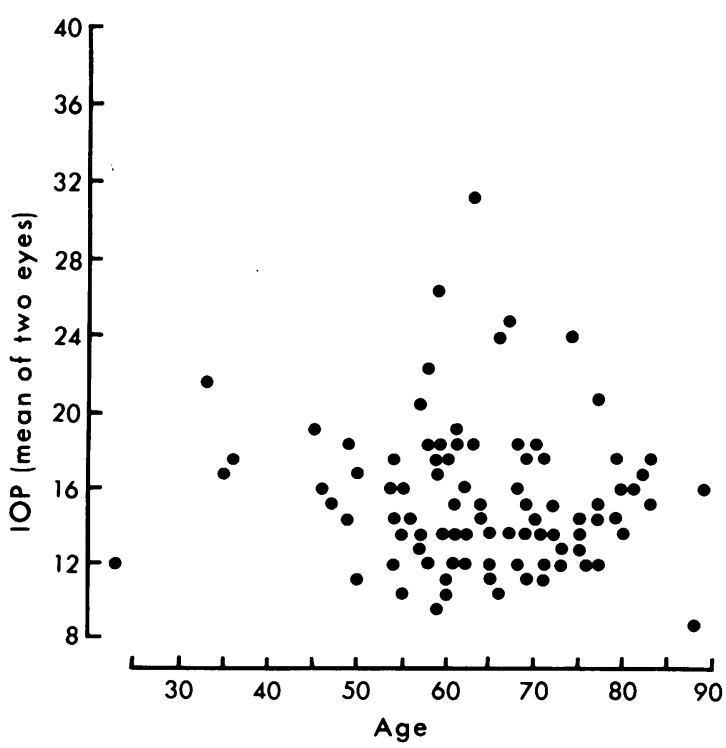

Fig. 2 IOP on age. Data scatter plot, stratified group. and of raised IOP. Indeed the adjusted residuals ${ }^{19}$ suggest a non-significant trend to early occurrence of raised IOP and a later occurrence of BP elevation. Analogous results were obtained in the two constituent groups of patients.

Figs. 2-4 display the raw data for the stratified group by means of scatter plots of IOP against age, systolic BP against age, and IOP against systolic BP.

The results of the univariate analyses are summarised in Table 3. A highly significant positive correlation was observed between systolic BP and age in all three groups, of the order of 0.34 to 0.44 . The diastolic pressure did not correlate with age nor with IOP. Numerically larger correlations were therefore observed between max IOP and systolic BP than between max IOP and mean BP.

While most correlations failed to reach significance, the max IOP-systolic BP correlation coefficients in the normal $(r=-0 \cdot 157)$ and $\mathrm{OH}(r=+0 \cdot 143)$ groups were statistically significantly different $(p=0.02)$. In the normal group age accounted for $\mathrm{R}^{2}=2.4 \%$ of the variation in max IOP. Again in the normal group systolic BP acounted for $\mathrm{R}^{2}=2.5 \%$ of the variation in max IOP.

In the stratified group, which had been formed to reflect the general population, the random mixing had resulted in a significant negative correlation between max IOP and systolic BP, accounting for slightly more than $4 \%$ of the variation in $\max$ IOP.

Multiple regressions of IOP and of max IOP on systolic BP and age were computed for each group. Table 4 summarises the results in terms of partial

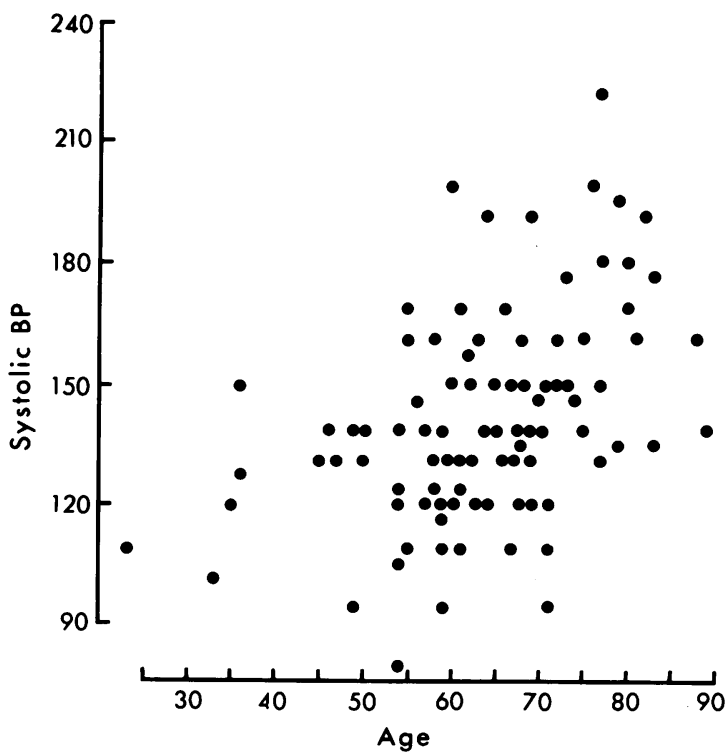

Fig. 3 Systolic BPon age. Data scatter plot, stratified group. 
Table 3 Paired correlation coefficients

\begin{tabular}{llrr}
\hline & Age & IOP & Max IOP \\
\hline Age & & $-0 \cdot 169(\mathrm{p}=0 \cdot 11)$ & $-0 \cdot 156(\mathrm{p}=0 \cdot 12)$ \\
Normals & & $-0 \cdot 035(\mathrm{p}=0 \cdot 67)$ & $-0 \cdot 0(2)(\mathrm{p}=0 \cdot 98)$ \\
OH & & $-0 \cdot 104(\mathrm{p}=0 \cdot 31)$ & $-0 \cdot 091(\mathrm{p}=0 \cdot 37)$ \\
Stratified & & & \\
Systolic BP & & $-0 \cdot 120(\mathrm{p}=0 \cdot 26)$ & $-0 \cdot 157(\mathrm{p}=0 \cdot 12)$ \\
Normals & $0 \cdot 446(\mathrm{p}=0 \cdot 0000)$ & $0 \cdot 102(\mathrm{p}=0 \cdot 22)$ & $0 \cdot 143(\mathrm{p}=0 \cdot 09)$ \\
OH & $0 \cdot 345(\mathrm{p}=0 \cdot 0000)$ & $-0 \cdot 184(\mathrm{p}=0 \cdot 06)$ & $-0 \cdot 206(\mathrm{p}=0 \cdot 04)$ \\
Stratified & $0 \cdot 441(\mathrm{p}=0 \cdot 0000)$ & & \\
\hline
\end{tabular}

$\mathrm{OH}=$ ocular hypertensives.

Table 4 Partial correlation coefficients

\begin{tabular}{llrr}
\hline Dependent & variable & \multicolumn{1}{l}{ IOP } & Max IOP \\
\hline Normals & Systolic BP & $-0.055(p=0.64)$ & $-0 \cdot 110(p=0.35)$ \\
& Age & $-0 \cdot 144(p=0.22)$ & $-0 \cdot 106(p=0.37)$ \\
OH & Systolic BP & $0 \cdot 164(p=0.06)$ & $0 \cdot 163(p=0.06)$ \\
& Age & $-0.048(p=0.59)$ & $-0.058(p=0.52)$ \\
Stratified & Systolic BP & $-0.172(p=0.13)$ & $-0.206(p=0.06)$ \\
& Age & $-0.028(p=0.80)$ & $-0.0004(p=0.99)$ \\
\hline
\end{tabular}

correlation coefficients. Max IOP showed a nearly significant $(p=0.06)$ negative partial correlation with systolic $B P$ in the stratified group and a nonsignificant partial correlation with age.

Analogous regression analyses were performed on younger persons only (60 years or less) in each of the groups. Results were much the same as reported above.

There was no indication that any of the three

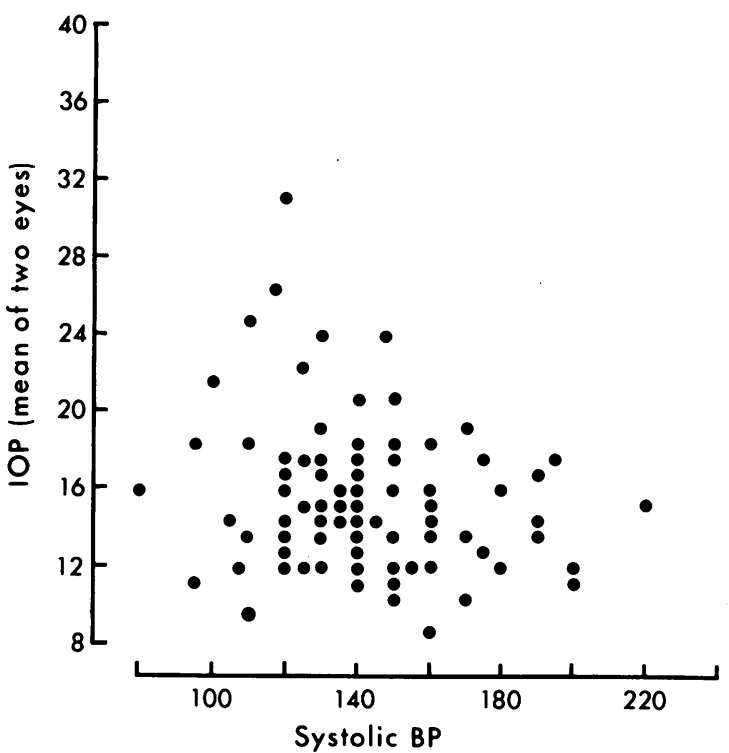

Fig. 4 IOP on systolic BP. Data scatter plot, stratified group. models (Fig. 1) provided an adequate description of the observations in the normal group, where the only correlation observed was between age and systolic BP. Model B could be considered to be descriptive of the ocular hypertensive group, if the level $p=0.06$ was taken as significant. In the stratified group, representing the general population, there was strong indication that model $\mathrm{C}$, the chain model, was adequate. Path analysis suggested a direct negative correlation of the order of -0.20 to -0.24 between IOP and systolic BP, and no direct correlation between IOP and age. Any apparent correlation in this direction was likely to be almost entirely due to the strong positive correlation between age and systolic BP. Indeed, if one lets $\mathrm{i}, \mathrm{j}$, and $\mathrm{g}$ in Galton's model above represent, respectively, age, max IOP, and systolic BP, one obtains from Table 3 , for the stratified group, that

$$
r_{i j}=-0 \cdot 091, r_{i g}=0 \cdot 441, r_{j g}=-0 \cdot 206,
$$

so that

$$
r_{i g} \times r_{j g}=-0.091=r_{i j} \text {, }
$$

with similar results for the mean IOP (mean of two eyes).

\section{Discussion}

Out data did not show on a cross-sectional basis that a raised systolic BP occurs at an earlier age than a raised IOP. Regression analyses on younger patients failed to confirm the postulated hypothesis that, after adjusting for BP, the age effect on IOP may be more apparent in younger people.

The results reported here may help to explain the inconsistencies found in the literature correlating IOP, BP, and age. Our analyses suggested different correlations in the normal group and in the ocular hypertensive group. When a random mixture of the two groups was formed in the proportions $93 \%$ normal pressures and 7\% ocular hypertensives, to reflect the composition of the general population, a distinct pattern of correlations was observed. While 
small sample sizes frequently led to only marginal statistical significance or non-significance, the relative sizes and signs of the coefficients formed a consistent pattern across the data. Moreover, absolute magnitudes were in close agreement with those given in the cited literature.

This work was aided by a grant from the Medical Research Council (68-1578).

\section{References}

1 Klein BE, Klein R. Intraocular pressure and cardiovascular risk variables. Arch Ophthalmol 1981; 99: 837-9.

2 Kahn HA, Milton RC. Alternative definitions of open angle glaucoma. Effect on prevalence and associations in the Framingham eye study. Arch Ophthalmol 1980; 98: 2172-7.

3 Leibowitz HM, Krueger DE, Maunder LR, et al. The Framingham Eye Study monograph: an ophthalmological and epidemiological study of cataract, glaucoma, diabetic retinopathy, macular degeneration and visual acuity in a general population of 2631 adults, 1973-1975. Surv Ophthalmol 1980; 24 (suppl): 335-610.

4 Leske MC, Podgor MJ. Intraocular pressure, cardiovascular risk variables, and visual field defects. Am J Epidemiol 1983; 118: 280-7.

5 Shiose Y, Kawase Y, Sato T, et al. Multivariate analysis on normal ocular tensions. Jpn J Clin Ophthalmol 1981; 35: 197-206.

6 Shiose Y. The aging effect on intraocular pressure in an apparently normal population. Arch Ophthalmol 1984; 102: 883-7.
7 Lu D, Liu X, Wang C. Analysis of intraocular pressure, cup disc ratio and systemic blood pressure for the prediction of prognosis in glaucoma patients. Eye Science 1985; 1: 77-80.

8 Duncan OD. Path analysis: sociological examples. Am J Sociol 은 1966; 72: 1-16.

9 Duncan OD. Structural equations models. New York: Academic Press, 1975.

10 Wright S. Path coefficients and path regressions: alternatives in complementary concepts? Biometrics 1960; 16: 423-45.

11 Wonnacott RJ, Wonnacott TH. Econometrics. New York: Wiley, 1970: 149-89.

12 Galton F. Hereditary genius: an enquiry into its laws and consequences. London: McMillan, 1869.

13 Spearman C. The proof and measurement of association between two things. Am J Psychol 1904; 15: 88-103.

14 Spearman C. 'General intelligence', objectively determined and measured. Am J Psychol 1904; 15: 201-93.

15 Drance SM, Schulzer M, Thomas B, Douglas GR. Multivariatc analysis in glaucoma. Use of discriminant analysis in predicting glaucomatous visual field damage. Arch Ophthalmol 1981; 99: 1019-22.

16 Drance SM, Schulzer M, Douglas GR, Sweency V. Usc of iN discriminant analysis. II. Identification of persons with glauco- $\mathrm{G}$ matous visual ficld defects. Arch Ophthalmol 1978; 96: 1571-3.

17 Armaly MF. Selective perimetry for glaucomatous defects in ocular hypertension. Arch Ophthalmol 1972; 87: 518-24.

18 Drance SM, Brais $P$, Fairclough M, et al. A screcning method for temporal visual defects in chronic simple glaucoma. Can J Ophthalmol 1972; 7: 428-9.

19 Everitt BS. The analysis of contingency tables. London: Chapman and Hall: New York: Wilcy: distributed in the USA by Halsted Press, 1977: 47.

Accepted for publication 30 May 1986.

$$
\text { N }
$$
ज 